\title{
Semi-global stabilization of linear systems subject to non-right invertible constraints
}

\author{
Ali Saberi * Anton A. Stoorvogel ${ }^{\dagger} \quad$ Guoyong Shi ${ }^{*} \quad$ Peddapullaiah Sannuti $^{\S}$
}

\begin{abstract}
Stabilization problems of linear systems with right invertible constraints have mostly been solved for both global and semi-global frameworks. Linear systems subject to non-right invertible constraints have completely different characteristics compared to those with right invertible constraints. This paper addresses the control problems of linear systems subject to non-right invertible constraints. This paper also expands further the available taxonomy of constraints. The new categorizations of constraints introduced reveal the difficulty in semi-global oriented control design for the non-right invertible constraints. Some new notions of stabilization are also introduced.
\end{abstract}

\section{Introduction}

Since all practical control systems are subject to realistic design constraints, the study of control problems with constraints is of a long standing interest. In particular, over the past ten years there has been renewed interest in such problems, mainly due to the introduction of some new perspectives. Global or semi-global stabilization occupies a prominent part of this research. A set of new design techniques have also been developed along this line. Two special issues $[1,11]$ account very well the progress made in the 90's.

Starting from Sontag and Sussmann [10], a number of research publications deal with systems subject to input saturation. In this regard some fundamental results have been established. It is known now that for linear stabilizable systems subject to input constraints, global or semi-global stabilization is possible if and only if open-loop poles do not have positive real parts [10]. Furthermore, it has become a well known fact that one must use nonlinear feedback for global stabilization [10] while linear feedback can achieve at most semi-global stabilization for systems containing a chain of integrators with order higher than two [4].

A new front-line of research on linear systems subject to constraints on both state and input variables is initiated recently by Saberi et al $[5,9]$. They consider global stabilization, and semi-global stabilization relative to an admissible set which is the set of all initial conditions such that the constraints are not violated. Also, utilization of state [5] as well as measurement feedback [9] are considered. For a class of constraints termed as right invertible constraints, the results presented in $[5,9]$ are complete in the sense that they develop the necessary and sufficient conditions under which the posed problems are solvable, and moreover they present as well the design algorithms of constructing appropriate controllers. The work of [5] has been extended recently by considering output regulation problems [7].

Our present work stems from [5,9], where a taxonomy of constraints plays a crucial role. A brief review of the taxonomy can be found in Section 2.1. We summarize the key results from [5] in term of the constraint taxonomy:

- Whether the constraints are right invertible or not, for both global and semi-global stabilization, it is necessary that the constraints are at most weakly nonminimum phase.

- For stabilizable systems with right invertible constraints, the constraints being at most weakly nonminimum phase is itself necessary and sufficient for semi-global stabilization.

- For stabilizable systems with right invertible constraints, the constraints being at most weakly nonminimum phase as well as being type one is necessary and sufficient for global stabilization.

An important aspect, for systems with right invertible constraints, is that the solvability conditions of the posed problems do not depend on the shape of the so called constraint set, i.e. the set within which the constrained output must lie. In other words, if the posed stabilization problems are solvable for one constraint set, they are also solvable for another constraint set. Also, as usual, one can use a linear control to achieve semi-global stabilization; however, in general one has to use nonlinear control laws for global stabilization.

It is important to recognize that whenever the constraints are only on the input and not on the state, they are always right invertible. Thus, the results of $[5,9]$ subsume all the available results regarding stabilization of linear systems subject to constraints only on inputs. Since constraints are

\footnotetext{
*School of Electrical Engineering and Computer Science, Washington State University, Pullman, WA 99164-2752, U.S.A. E-mail: saberi@eecs.wsu.edu.

$\dagger$ Department of Mathematics, and Computing Science, Eindhoven Univ. of Technology, P.O. Box 513, 5600 MB Eindhoven, The Netherlands. E-mail: A.A.Stoorvogel@tue.nl. Department of Information Technology and Systems, Delft Univ. of Technology, P.O. Box 5031, 2600 GA Delft, The Netherlands

Electrical Engineering Department, University of Washington, Seattle, WA 98195, U.S.A. E-mail: gshi@ee.washington.edu

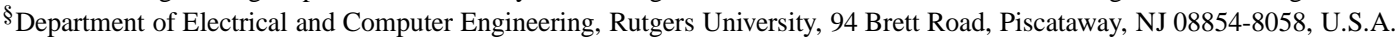
E-mail: sannuti@ece.rutgers.edu
} 
always right invertible when they are only on inputs, nonright invertible constraints, whenever they arise, are inherently due to state constraints. For systems with non-right invertible constraints, although [5,9] present some partial results, the work is far from being complete. Nevertheless, partial results of [5,9] provide an understanding of the issues involved, and thereby identify the complex issues, challenges, and the difficulties one faces with non-right invertible constraints. One of the fundamental difficulties in dealing with non-right invertible constraints is that the solvability conditions for the posed stabilization problems depend not only on the structural properties of the mapping from the input to the constrained output but also on the shape of the constraint set within which the constrained output must lie. Because of such difficulties, stabilization problems of linear systems with non-right invertible constraints remained as open research problems for the last two or three years. In this paper, we confront the issues and challenges raised in [5,9], and more or less resolve most of them quite satisfactorily. In particular, we present here necessary and sufficient conditions for the solvability of the semi-global stabilization problem. The solvability conditions developed here are straightforward to check for the common case when the constraint set is a polytope. We illustrate these results on a DC motor example. Also, the above mentioned results motivate us to introduce new notions of stabilization with some reasonably large domain of attraction, but not necessarily in the sense of semi-global stabilization. Moreover, our results lead us to expand the taxonomy of constraints developed earlier so that various issues that arise in connection with non-right invertible constraints can easily be revealed and channelized.

Due to the space limitation, all proofs are omitted in this proceedings version.

\section{Preliminaries and problem formu- lations}

Consider a linear continuous-time system,

$$
\Sigma: \begin{cases}\dot{x}=A x+B u & x \in \mathbb{R}^{n}, u \in \mathbb{R}^{m} \\ y=C_{y} x+D_{y} u, & y \in \mathbb{R}^{\ell} \\ z=C_{z} x+D_{z} u & z \in \mathbb{R}^{p}\end{cases}
$$

where $x$ is the state, $u$ is the input, $y$ is the measured output, and $z$ is the constrained output (see Figure 1). The constrained output is subject to the constraint

$$
z(t) \in \mathcal{S}, \quad \forall t \geqslant 0
$$

where the set $\& \subset \mathbb{R}^{p}$ is a priori given and is referred to as the constraint set. The following assumption on the constraint set $\&$ and the constrained output is used throughout the paper.

\section{Assumption 2.1}

(i) The set 8 is compact, convex, and contains 0 as an interior point.

(ii) $C_{z}^{T} D_{z}=0$ and $s=\left(\& \cap \operatorname{im} C_{z}\right)+\left(\& \cap \operatorname{im} D_{z}\right)$.

Definition 2.2 Let the system (2.1) and a constraint set $\&$ be given. We define

$$
\mathcal{A}(\delta):=\left\{x \in \mathbb{R}^{n} \mid \exists u \text { such that } C_{z} x+D_{z} u \in \delta\right\}
$$

as the admissible set of initial conditions.

Remark. In view of Assumption 2.1, we observe that the admissible set $\mathcal{A}(\&)$ can be equivalently written as

$$
\mathcal{A}(\delta):=\left\{x \in \mathbb{R}^{n} \mid C_{z} x \in \delta\right\} .
$$

We shall use the following two concepts in this paper.

Definition 2.3 Consider the given system $\Sigma$ as in (2.1) together with a constraint set \& satisfying Assumption 2.1. Then, the constrained recoverable region $\mathcal{R}_{C}(\Sigma, 8)$ of this system is the set of all initial states $x(0) \in \mathcal{A}(\Sigma, \&)$ for which there exists a control input $u$ such that $x(t) \rightarrow 0$ as $t \rightarrow \infty$ while $z(t) \in \&$ for all $t \geqslant 0$.

\section{Definition 2.4 Given the system}

$$
\begin{aligned}
& \dot{x}=f(x), \quad x(0)=x_{0} \\
& z=h(x)
\end{aligned}
$$

with $x(t) \in \mathbb{R}^{n}$ and $z(t) \in \mathbb{R}^{p}$. Let a constraint set $\& \subset \mathbb{R}^{p}$ be given and let 0 be a stable equilibrium point of the system.

The constrained domain of attraction $\mathcal{R}_{A}(\Sigma, 8)$ consists of all $x_{0} \in \mathbb{R}^{n}$ for which $x(t) \rightarrow 0$ as $t \rightarrow \infty$ while $z(t) \in \delta$ for all $t>0$.

When we state that a controller achieves a constrained domain of attraction $\mathcal{R}_{A}(\Sigma, 8)$ we mean that the resulting closed loop system has 0 as a stable equilibrium point and $\mathcal{R}_{A}(\Sigma, 8)$ is the constrained domain of attraction of the closed loop system.

We mainly deal with two semi-global stabilization problems as described below:

Problem 2.5 Consider the system (2.1) along with a constraint set $\& \subset \mathbb{R}^{p}$ satisfying Assumption 2.1. Constrained semi-global stabilization via state feedback is concerned with finding a family of state feedbacks (possibly nonlinear and time-varying) of the form $u(t)=f(x(t), t)$ such that, for any a priori given compact set $W$ contained in the interior of $\mathcal{A}(\&)$, there exists a state feedback in this family such that the equilibrium point $x=0$ of the closed-loop system is asymptotically stable with $\mathcal{W}$ contained in the constrained domain of attraction. 


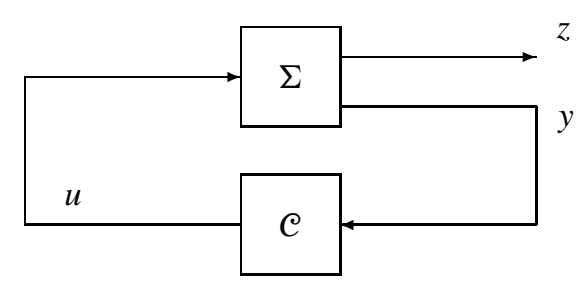

Figure 1: Closed-loop system subject to constrained output

Problem 2.6 Consider a system of the form (2.1) with a constraint set $\& \subset \mathbb{R}^{p}$ satisfying Assumption 2.1. Constrained semi-global stabilization via measurement feedback is concerned with finding (if possible) a family of measurement feedbacks of the form

$$
\left\{\begin{array}{l}
\dot{v}=g(v, y, t), \quad v \in \mathbb{R}^{q} \\
u=h(v, y, t)
\end{array}\right.
$$

such that for any compact set $\mathcal{X} \subset$ int $\mathcal{A}(\delta)$ and any compact set $\mathcal{V} \subset \mathbb{R}^{q}$ there exists a measurement feedback in this family such that the following conditions hold:

(i) The equilibrium point $(x, v)=(0,0)$ of the closedloop system is asymptotically stable with $\mathcal{X} \times \mathcal{V}$ contained in its region of attraction.

(ii) For any $(x(0), v(0)) \in \mathcal{X} \times \mathcal{V}$, we have $z(t) \in \&$ for all $t \geqslant 0$.

The main objective of this paper is to establish the necessary and sufficient conditions under which the above problems are solvable.

\subsection{Taxonomy of Constraints}

For the systems of the form $\Sigma$ given by (2.1), we briefly review here the taxonomy of constraints that has emerged from the study of stabilization problems of such systems [5], and also add one additional component to this taxonomy which arises from the results presented in this paper. Let $\Sigma_{z}$ denote the subsystem quadruple characterized by the quadruple $\left(A, B, C_{z}, D_{z}\right)$. The right invertibility, the location of invariant zeros, and the order of infinite zeros of $\Sigma_{z}$ dictate the solvability conditions of some constrained stabilization problems.

The first category in the taxonomy of constraints is based on whether the system $\Sigma_{z}$ is right invertible or not.

Definition 2.7 The constraints are said to be

- right invertible constraints if the system $\Sigma_{z}$ characterized by $\left(A, B, C_{z}, D_{z}\right)$ is right invertible.

- non-right invertible constraints if the system $\Sigma_{z}$ is non-right invertible.

In line with the above categorization, a new categorization based on the results of this paper is introduced here. This categorization requires a specific structure of the system $\Sigma_{z}$ when expressed in terms of a special coordinate basis as presented in Appendix A.

Definition 2.8 Given the system $\Sigma_{z}$ characterized by the quadruple $\left(A, B, C_{z}, D_{z}\right)$, convert it into the special coordinate basis (see Appendix A).

- The constraints are said to be weakly non-rightinvertible if the system is not right invertible and the matrix $C_{b}$ is injective.

- The constraints are said to be strongly non-rightinvertible if the system is not right invertible and the matrix $C_{b}$ is not injective.

The invariant zeros of the system $\Sigma_{z}$ are labeled as constraint invariant zeros of the plant, and our second category of constraints is based on the location of these constraint invariant zeros. In the following definition, $\mathbb{C}^{-}, \mathbb{C}^{0}$, and $\mathbb{C}^{+}$ denote respectively the set of complex numbers with negative real parts, zero real parts, and positive real parts.

Definition 2.9 The constraints are said to be

- minimum phase constraints if all the constraint invariant zeros are in $\mathbb{C}^{-}$.

- weakly minimum phase constraints if all the constraint invariant zeros are in $\mathbb{C}^{-} \cup \mathbb{C}^{0}$ with the restriction that at least one such constraint invariant zero is in $\mathbb{C}^{0}$ and any such constraint invariant zero in $\mathbb{C}^{0}$ is simple.

- weakly non-minimum phase constraints if all the constraint invariant zeros are in $\mathbb{C}^{-} \cup \mathbb{C}^{0}$ and at least one constraint invariant zero in $\mathbb{C}^{0}$ is not simple.

- at most weakly non-minimum phase constraints if all the constraint invariant zeros are in $\mathbb{C}^{-} \cup \mathbb{C}^{0}$.

- strongly non-minimum phase constraints if one or more of the constraint invariant zeros are in $\mathbb{C}^{+}$.

The third categorization is based on the order of the infinite zeros of the system $\Sigma$, which are labeled as the constraint infinite zeros of the plant.

Definition 2.10 The constraints are said to be type one constraints if the order of all constraint infinite zeros is less than or equal to one. 


\section{Main results}

In order to present our main result we need to define the following. Consider the system (2.1) along with a constraint set $s \subset \mathbb{R}^{p}$ satisfying Assumption 2.1. We transform the system $\Sigma_{z}$ characterized by the quadruple $\left(A, B, C_{z}, D_{z}\right)$ into the special coordinate basis according to Appendix A. We define $\bar{s}=T_{z}^{-1} \&$ and

$$
\overline{\mathcal{A}}(\bar{\S}):=\left\{x_{b} \in \mathbb{R}^{n_{b}} \mid \exists \zeta \text { such that }\left(\begin{array}{c}
C_{b} x_{b} \\
\zeta
\end{array}\right) \in \bar{s}\right\} .
$$

Also, let $N_{\xi} \subset \mathbb{R}^{n}$ be given by

$$
N_{\xi}:=\left\{\eta \mid\|\eta\|=1 \text { and }\left\langle\xi^{\prime}-\xi, \eta\right\rangle \leqslant 0, \forall \xi^{\prime} \in \overline{\mathcal{A}}(\bar{\S})\right\} .
$$

Then, for any $\xi \in \partial \overline{\mathcal{A}}(\bar{\S})$, we define

$$
\begin{aligned}
T_{\xi}:= & \left\{\mu \in \mathbb{R}^{n_{b}} \mid \mu=A_{0} \xi+K_{b 2} \zeta \text { with } \zeta \in \mathbb{R}^{m_{0}+\ell}\right. \\
& \text { such that } \left.\left(\begin{array}{c}
C_{b} \xi \\
\zeta
\end{array}\right) \in \bar{\jmath} \text { and }\langle\mu, \eta\rangle \leqslant 0, \forall \eta \in N_{\xi}\right\}
\end{aligned}
$$

where $A_{0}=A_{b}+K_{b b} C_{b}$, and where $K_{b 2}$ and $K_{b b}$ are as defined in Appendix A. Note that $N_{\xi}$ is the collection of normal vectors yielding hyperplanes through the point $\xi$ which are tangent planes to the set $\overline{\mathcal{A}}(\bar{\delta})$. On the other hand, $T_{\xi}$ is the collection of directions a trajectory starting in $\xi$ can take pointing inside or alongside the admissible set $\overline{\mathcal{A}}(\bar{\delta})$.

Next, we present our main results.

Theorem 3.1 Consider the system (2.1) along with a constraint set $\& \subset \mathbb{R}^{p}$ satisfying Assumption 2.1. Let the system $\Sigma_{z}$ characterized by the quadruple $\left(A, B, C_{z}, D_{z}\right)$ be transformed into the special coordinate basis according to Appendix A. Then, constrained semi-global stabilization via state feedback as defined in Problem 2.5 is solvable if and only if:

(i) $(A, B)$ stabilizable,

(ii) The constraints are at most weakly non-minimumphase,

(iii) The constraints are either right invertible or weakly non-right invertible; and

(iv) If the constraints are not right invertible, then the set $T_{\xi}$ is nonempty for all $\xi \in \partial \bar{A}(\bar{\delta})$.

The first three conditions of Theorem 3.1 are on the structural properties of $\Sigma_{z}$, where as the last condition is on the constraint set $\$$. We note that in nearly all constrained systems the constrained set $\delta$ can be assumed to be polyhedral. In that case, a major simplification in the above necessary and sufficient conditions can be established as given by the following theorem.
Theorem 3.2 Consider the system (2.1) along with a constraint set $\& \subset \mathbb{R}^{p}$ satisfying Assumption 2.1. Let the system $\Sigma_{z}$ characterized by the quadruple $\left(A, B, C_{z}, D_{z}\right)$ be transformed into the special coordinate basis according to Appendix A. Then we have:

(i) If the set $\&$ is polyhedral, then the set $\overline{\mathcal{A}}(\overline{\mathcal{S}})$ is a polyhedral as well, and moreover, it has no more corner points than the set 8 .

(ii) If the set $\&$ is polyhedral and $T_{\xi}$ is nonempty for all corner points of $\overline{\mathcal{A}}(\overline{\mathcal{S}})$, then it is nonempty for all $\xi \in \partial \overline{\mathcal{A}}(\bar{\S})$.

The above theorem is crucial in that it establishes that we need to check only a finite number of conditions to verify the condition (iv) of Theorem 3.1.

As we said earlier, the only condition in Theorem 3.1 that depends on the structure of the constraint set $\&$ is condition (iv). It is interesting to note that the only case for which condition (iv) is satisfied for any constraint set $\&$ satisfying Assumption 2.1 is when the matrix $A_{0}$ has a special structure as established in the following theorem.

Theorem 3.3 Consider the system $\Sigma$ given by (2.1) with $(A, B)$ stabilizable. Assume that the system $\Sigma_{z}$ characterized by the quadruple $\left(A, B, C_{z}, D_{z}\right)$ is weakly non-right invertible. Also, let $\Sigma_{z}$ be transformed into the special coordinate basis according to Appendix A. Then, condition (iv) of Theorem 3.1 is satisfied for all constraint sets \& satisfying Assumption 2.1 if and only if $A_{0}=\alpha I$ with $\alpha \leqslant 0$.

Remark. Theorem 3.3 recovers Theorem 3.1 of [5] as a special case.

The following theorem pertains to measurement feedback.

Theorem 3.4 Consider the plant $\Sigma$ as given by (2.1) and a constraint set \& that satisfies Assumption 2.1. Let the system $\Sigma_{z}$ characterized by the quadruple $\left(A, B, C_{z}, D_{z}\right)$ be transformed into the special coordinate basis according to Appendix A. Then, the constrained semi-global stabilization problem via measurement feedback as defined in Problem 2.6 is solvable if the following conditions hold:

(i) $(A, B)$ is stabilizable,

(ii) The constraints are at most weakly non-minimum phase,

(iii) The constraints are either right invertible or weakly non-right invertible,

(iv) If the constraints are not right invertible, then the set $T_{\xi}$ is nonempty for all $\xi \in \partial \bar{A}(\bar{\S})$,

(v) The pair $\left(C_{y}, A\right)$ is observable.

Moreover, the first four conditions are necessary. 


\section{Planar systems with full state and input constraints}

Consider a second order controllable single-input system of the form,

$$
\left\{\begin{array}{l}
\dot{x}=A x+B u \\
z=\left(\begin{array}{l}
I \\
0
\end{array}\right) x+\left(\begin{array}{l}
0 \\
1
\end{array}\right) u
\end{array}\right.
$$

with a constraint $z(t) \in \delta$ where we assume here that $\delta$ is a hypercube:

$$
\left\{\left(\begin{array}{l}
z_{1} \\
z_{2} \\
z_{3}
\end{array}\right) \in \mathbb{R}^{3}|| z_{1}\left|\leqslant \alpha_{1}, \quad\right| z_{2}\left|\leqslant \alpha_{2}, \quad\right| z_{3} \mid \leqslant \alpha_{3}\right\} .
$$

It is easy to see that the constraints on this system are not right invertible.

The DC motor example as in Kosut [3] is a special case where

$$
A=\left(\begin{array}{cc}
0 & 1 \\
-a_{1} & -a_{2}
\end{array}\right), \quad B=\left(\begin{array}{l}
0 \\
b
\end{array}\right),
$$

and where $a_{1}=3575, a_{2}=333$, and $b=305555$. Note that these values are obtained from experiments. Also, $x_{1}$ is the motor shaft angular velocity $(\mathrm{rad} / \mathrm{s})$ and $x_{2}$ is the acceleration $\left(\mathrm{rad} / \mathrm{s}^{2}\right)$. Moreover, for this specific example,

$$
\alpha_{1}=8.75 \mathrm{rad} / \mathrm{s}, \quad \alpha_{2}=825 \mathrm{rad} / \mathrm{s}^{2}, \quad \alpha_{3}=1 \mathrm{~V} .
$$

For a general second order system as defined above in (4.1), we want to check whether semi-global regulation is possible.

We obtain the following lemma.

Lemma 4.1 Given the second order system in (4.1) with constraint set $\&$ as in (4.2). Let

$$
A=\left(\begin{array}{ll}
A_{11} & A_{12} \\
A_{21} & A_{22}
\end{array}\right), \quad B=\left(\begin{array}{l}
B_{1} \\
B_{2}
\end{array}\right) .
$$

Without loss of generality we assume both $B_{1}$ and $B_{2}$ are both nonnegative (this can always be achieved via a state space transformation replacing $x_{1}$ by $-x_{1}$ and/or $x_{2}$ by $\left.-x_{2}\right)$. Then constrained semi-global stabilization is possible if and only if:

$$
\begin{aligned}
& A_{11} \alpha_{1}+\left|A_{12} \alpha_{2}\right|-B_{1} \alpha_{3} \leqslant 0 \\
& A_{22} \alpha_{2}+\left|A_{21} \alpha_{1}\right|-B_{2} \alpha_{3} \leqslant 0, \\
& B_{2}\left(A_{12} \alpha_{2}-A_{11} \alpha_{1}\right) \geqslant B_{1}\left(A_{22} \alpha_{2}-A_{21} \alpha_{1}\right) .
\end{aligned}
$$

Returning to the special case of the DC motor example, we can easily check that the conditions in Lemma 4.1 are not satisfied. Although the system has no constraint invariant zeros and is weakly non-right invertible, the algebraic conditions of the lemma fail. Also, although in this example the open loop system is stable, the regulation speed of $x_{1}$ is not fast enough. Kosut used the circle criterion to derive the gain parameters for fast speed regulation, that is, to regulate the speed $x_{1}$ to zero as fast as possible. His derivation leads to the feedback gain parameters $f_{2}=0.36$ and $f_{1}=\gamma f_{2}=420 * 0.36=151.2$ by taking $\gamma=420$.

The following figures depict some simulation results. For visualization purpose, we plot a set of state trajectories in each simulation. Also for better visualization, the $x_{2}$ axis is scaled by $x_{2} / 100$.

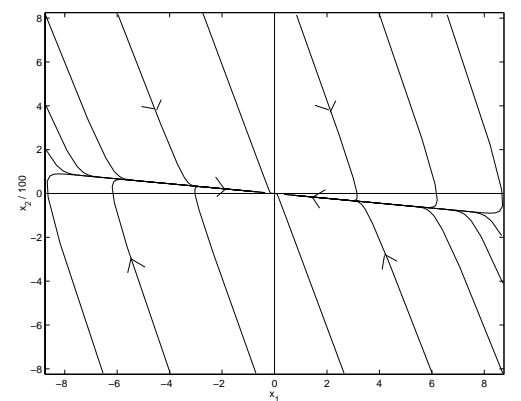

The above figure shows the state trajectories without control. Note that the DC motor is open loop stable, but the domain of attraction does not cover the whole admissible set. Also, the way $x_{1}$ goes to zero is not fast enough.

The next figure shows the state trajectories by applying Kosut's design derived from the circle criterion,

$$
u=\sigma\left(-151.2 x_{1}-0.36 x_{2}\right) .
$$

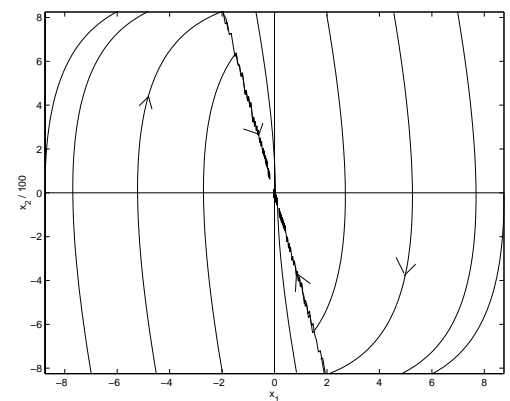

The simulation results in the second figure might encourage us to predict the possibility of semi-global stabilization relative to an admissible set. However, as noted before, the results of this paper show that this is not possible.

\section{Conclusion}

This paper concludes a line of research establishing the necessary and sufficient conditions for the solvability of semiglobal constrained stabilization problem. The main open problem is to establish the solvability conditions for the global case. 
It is interesting to note that in the heart of the development of this paper, we make a subset of the state space invariant by feedback which by itself is a line of research studied extensively (see for instance the survey paper [2]). The main difference here however is that in a semi-global framework of stabilization we need to identify conditions for being able to find a set arbitrary close to the admissible set which can be made invariant. This required us to develop new tools as reported in this paper and our earlier work that lead to this paper.

\section{A Special coordinate basis}

We recall a special coordinate basis (scb) $[6,8]$. For a general linear system $\Sigma$ in (2.1), by utilizing the quadruple $\left(A, B, C_{z}, D_{z}\right)$, one can choose appropriate coordinates in the state, input, and output spaces, say

$$
x=T_{x} \bar{x}, \quad u=T_{u} \bar{u}, \quad z=T_{z} \bar{z},
$$

where $T_{x}, T_{u}$, and $T_{z}$ are the respective transformation matrices, so that the system (2.1) takes the following form

$$
\bar{\Sigma}:\left\{\begin{array}{l}
\dot{x}_{a}=A_{a} x_{a}+K_{a} \bar{z}, \\
\dot{x}_{b}=A_{b} x_{b}+K_{b} \bar{z}, \\
\dot{x}_{c}=A_{c} x_{c}+B_{c}\left(u_{c}+H_{a} x_{a}\right)+K_{c} \bar{z}, \\
\dot{x}_{d}=A_{d} x_{d}+B_{d}\left(u_{d}+G_{a} x_{a}+G_{b} x_{b}+G_{c} x_{c}+G_{d} x_{d}\right) \\
y=K_{d} \bar{z}, \\
\bar{z}=\left(\begin{array}{c}
z_{b} \bar{x}+D_{y} \bar{u} \\
z_{0} \\
z_{d}
\end{array}\right)=\left(\begin{array}{c}
C_{b} x_{b} \\
u_{0} \\
C_{d} x_{d}
\end{array}\right),
\end{array}\right.
$$

where $\bar{x}^{\mathrm{T}}=\left(x_{a}^{\mathrm{T}}, x_{b}^{\mathrm{T}}, x_{c}^{\mathrm{T}} x_{d}^{\mathrm{T}}\right)$ and $\bar{u}^{\mathrm{T}}=\left(u_{0}^{\mathrm{T}} u_{c}^{\mathrm{T}}, u_{d}\right)$ are the state and input in the new coordinate system. Here, $x_{a} \in \mathbb{R}^{n_{a}}, x_{b} \in \mathbb{R}^{n} b, x_{c} \in \mathbb{R}^{n_{c}}$, and $x_{d} \in \mathbb{R}^{n} d$, with $n_{a}+n_{b}+n_{c}+n_{d}=n$, and $u_{0} \in \mathbb{R}^{m_{0}}, u_{c} \in \mathbb{R}^{m_{c}}$, and $u_{d} \in \mathbb{R}^{\ell}$, with $m_{0}+m_{c}+\ell=m$. Also, $z_{0} \in \mathbb{R}^{m_{0}}, z_{b} \in \mathbb{R}^{m_{b}}$, and $z_{d} \in \mathbb{R}^{\ell}$, with $m_{0}+m_{b}+\ell=p$. Moreover, the matrix pair $\left(C_{b}, A_{b}\right)$ is observable and the matrix pair $\left(A_{c}, B_{c}\right)$ is controllable.

Furthermore,

$$
\begin{aligned}
A_{d} & =\text { block } \operatorname{diag}\left[A_{1}, \cdots, A_{\ell}\right], \quad B_{d}=\operatorname{block} \operatorname{diag}\left[B_{1}, \cdots, B_{\ell}\right], \\
C_{d} & =\text { block diag }\left[C_{1}, \cdots, C_{\ell}\right],
\end{aligned}
$$

where $A_{i}=\left(0, e_{1}, \cdots, e_{k_{i}-1}\right) \in \mathbb{R}^{k_{i} \times k_{i}}, B_{i}=e_{k_{i}} \in \mathbb{R}^{k_{i} \times 1}$, and $C_{i}=e_{1}^{\mathrm{T}} \in \mathbb{R}^{1 \times k_{i}}$ for $i=1, \cdots, \ell$, where $e_{i}$ is the $i$ th standard basis vector with appropriate dimension, and $\sum_{i=1}^{\ell} k_{i}=n_{d}$. Also, the eigenvalues of $A_{a}$ are the invariant zeros of the subsystem $\Sigma_{z}$. Moreover, $\Sigma$ is right invertible if and only if $x_{b}$ is non-existent while $\Sigma$ is left invertible if and only if $x_{c}$ is non-existent.

For the purpose of this specific paper it is convenient to introduce

$$
\zeta=\left(\begin{array}{l}
z_{0} \\
z_{d}
\end{array}\right),
$$

and decompose $K_{b}$ and $K_{a}$ to be compatible with the decomposition of

$$
\begin{gathered}
\bar{z}=\left(\begin{array}{c}
z_{b} \\
\zeta
\end{array}\right), \\
K_{b}=\left(\begin{array}{ll}
K_{b b} & K_{b 2}
\end{array}\right), \text { and } \\
K_{a}=\left(\begin{array}{ll}
K_{a a} & K_{a 2}
\end{array}\right) .
\end{gathered}
$$

\section{References}

[1] D.S. Bernstein And A.N. Michel, Guest Eds., Special Issue on saturating actuators, Int. J. Robust \& Nonlinear Control, 5(5), 1995.

[2] F. BLANCHINI, "Set invariance in control", Automatica, 35(11), 1999, pp. 1747-1769.

[3] R.L. Kosut, "Design of linear systems with saturating linear control and bounded states", IEEE Trans. Aut. Contr., 28(1), 1983, pp. 121-124.

[4] Z. LiN AND A. SABERI, "Semi-global exponential stabilization of linear systems subject to "input saturation" via linear feedbacks", Syst. \& Contr. Letters, 21(3), 1993, pp. 225-239.

[5] A. Saberi, J. Han, and A.A. Stoorvogel, "Constrained stabilization problems for linear plants", Automatica, 38(4), 2002, pp. 639-654.

[6] A. SABERI AND P. SANnUti, "Squaring down of nonstrictly proper systems", Int. J. Contr., 51(3), 1990, pp. 621-629.

[7] A. Saberi, A.A. Stoorvogel, G. Shi, AND P. SANNUTI, "Output regulation of linear plants subject to state and input constraints", in Actuator saturation control, G. Grigoriadis V. Kapila, ed., vol. 12 of Control Engineering Series, Marcel Dekker, 2002, pp. 189-226.

[8] P. Sannuti And A. SABeri, "Special coordinate basis for multivariable linear systems - finite and infinite zero structure, squaring down and decoupling", Int. J. Contr., 45(5), 1987, pp. 1655-1704.

[9] G. Shi, A. SABEri, A.A. Stoorvogel, AND P. SANNUTI, "Semi-global stabilization and output regulation of constrained linear plants via measurement feedback", Int. J. Contr., 75(18), 2002, pp. 1525-1538.

[10] E.D. Sontag AND H.J. Sussmann, "Nonlinear output feedback design for linear systems with saturating controls", in Proc. 29th CDC, Honolulu, 1990, pp. 3414-3416.

[11] A. Saberi And A.A. Stoorvogel, Guest Eds., Special Issue on control problems with constraints, Int. J. Robust \& Nonlinear Control, 9(10), 1999. 